\title{
Stability and Hopf Bifurcation in a Modified Holling-Tanner Predator-Prey System with Multiple Delays
}

\author{
Zizhen Zhang, ${ }^{1,2}$ Huizhong Yang, ${ }^{1}$ and Juan Liu ${ }^{3}$ \\ ${ }^{1}$ Key Laboratory of Advanced Process Control for Light Industry of Ministry of Education, \\ Jiangnan University, Wuxi 214122, China \\ ${ }^{2}$ School of Management Science and Engineering, Anhui University of Finance and Economics, \\ Bengbu 233030, China \\ ${ }^{3}$ Department of Science, Bengbu College, Bengbu 233030, China \\ Correspondence should be addressed to Huizhong Yang, yanghzjiangnan@163.com
}

Received 9 August 2012; Revised 17 September 2012; Accepted 4 October 2012

Academic Editor: Kunquan Lan

Copyright (C) 2012 Zizhen Zhang et al. This is an open access article distributed under the Creative Commons Attribution License, which permits unrestricted use, distribution, and reproduction in any medium, provided the original work is properly cited.

A modified Holling-Tanner predator-prey system with multiple delays is investigated. By analyzing the associated characteristic equation, the local stability and the existence of periodic solutions via Hopf bifurcation with respect to both delays are established. Direction and stability of the periodic solutions are obtained by using normal form and center manifold theory. Finally, numerical simulations are carried out to substantiate the analytical results.

\section{Introduction}

Predator-prey dynamics has long been and will continue to be of interest to both applied mathematicians and ecologists due to its universal existence and importance [1, 2]. Many population models investigating the dynamic relationship between predators and their preys have been proposed and studied. For example, Lotka-Volterra model [3-5], LeslieGower model [6-10], and Holling-Tanner model [11-16]. Among these widely used models, Holling-Tanner model plays a special role in view of the interesting dynamics it possesses. Holling-Tanner model for predator-prey interaction is governed by the following nonlinear coupled ordinary differential equations:

$$
\begin{gathered}
\frac{d X}{d T}=r X\left(1-\frac{X}{K}\right)-\frac{m X Y}{a+X} \\
\frac{d Y}{d T}=Y\left[s\left(1-h \frac{Y}{X}\right)\right]
\end{gathered}
$$


where $X$ and $Y$ denote the population densities of prey species and predator species at time $T$, respectively. The first equation in system (1.1) shows that the prey grows logistically with the carrying capacity $K$ and the intrinsic growth rate $r$ in the absence of the predator. And the growth of the prey is hampered by the predator at a rate proportional to the functional response $m X /(a+X)$ in the presence of the predator. The second equation shows that the predator consumes the prey according to the functional response $m X /(a+X)$ and grows logistically with the intrinsic growth rate $s$ and carrying capacity $X / h$ proportional to the number of the prey. The parameter $m$ denotes the maximal predator per capita consumption rate. $a$ is a saturation value; it corresponds to the number of prey necessary to achieve one half the maximum rate $m$. The parameter $h$ denotes the number of prey required to support one predator at equilibrium when $y$ equals $X / h$.

Recently, there has been considerable interest in predator-prey systems with the Beddington-DeAngelis functional response. And it has been shown that the predator-prey systems with the Beddington-DeAngelis functional response have rich but biologically reasonable dynamics. For more details about this functional response one can refer to [1721]. Zhang [16], Lu and Liu [22] considered the following modified Holling-Tanner delayed predator-prey system:

$$
\begin{gathered}
\frac{d X}{d T}=r X\left(1-\frac{X}{K}\right)-\frac{\alpha X Y}{a+b X+c Y}, \\
\frac{d Y}{d T}=Y\left[s\left(1-h \frac{Y(t-\bar{\tau})}{X(t-\bar{\tau})}\right)\right],
\end{gathered}
$$

where $\bar{\tau}$ is incorporated in the negative feedback of the predator density. $\alpha X Y /(a+b X+c Y)$ is the Beddington-DeAngelis functional response. The parameters $\alpha, a, b$, and $c$ are assumed to be positive. $\alpha$ is the maximum value at which per capita reduction rate of the prey can attain. $a$ measures the extent to which environment provides protection to the prey. $b$ describes the effect of handling time on the feeding rate. $c$ describes the magnitude of interference among predators. Zhang [16] investigated the local Hopf bifurcation of system (1.2). Lu and Liu [22] proved that system (1.2) is permanent under some conditions and obtained the sufficient conditions of local and global stability of system (1.2). Since both the species are growing logistically, it is reasonable to assume delay in prey species as well. Based on this consideration, we incorporate the negative feedback of the prey density into system (1.2) and obtain the following system:

$$
\begin{gathered}
\frac{d X}{d T}=r X\left(1-\frac{X\left(T-T_{1}\right)}{K}\right)-\frac{\alpha X Y}{a+b X+c Y^{\prime}} \\
\frac{d Y}{d T}=Y\left[s\left(1-h \frac{Y\left(T-T_{2}\right)}{X\left(T-T_{2}\right)}\right)\right],
\end{gathered}
$$

where $T_{1}$ and $T_{2}$ are the feedback time delays of the prey density and the predator density respectively. Let $X=K x, Y=(r K / \alpha) y, t=r T, \tau_{1}=r T_{1}, \tau_{2}=r T_{2}$, system (1.3) can be 
transformed into the following nondimensional form:

$$
\begin{gathered}
\frac{d x}{d t}=x\left(1-x\left(t-\tau_{1}\right)\right)-\frac{x y}{a_{1}+b x+c_{1} y}, \\
\frac{d y}{d t}=y\left[\delta-\beta \frac{y\left(t-\tau_{2}\right)}{x\left(t-\tau_{2}\right)}\right]
\end{gathered}
$$

where $a_{1}=a / K, c_{1}=c r / \alpha, \delta=s / r, \beta=s h / \alpha$ are the non-dimensional parameters and they are positive.

The main purpose of this paper is to consider the effect of multiple delays on system (1.4). The local stability of the positive equilibrium and the existence of Hopf bifurcation are investigated. By employing normal form and center manifold theory, the direction of Hopf bifurcation and the stability of the bifurcating periodic solutions are determined. Finally, some numerical simulations are also included to illustrate the theoretical analysis.

\section{Local Stability and the Existence of Hopf Bifurcation}

In this section, we study the local stability of each of feasible equilibria and the existence of Hopf bifurcation at the positive equilibrium. Obviously, system (1.4) has a unique boundary equilibrium $E_{1}(1,0)$ and a unique positive equilibrium $E_{*}\left(x_{*}, y_{*}\right)$, where

$$
x_{*}=\frac{-\left[\left(a_{1}-b\right) \beta+\left(1-c_{1}\right) \delta\right]+\sqrt{\left[\left(a_{1}-b\right) \beta+\left(1-c_{1}\right) \delta\right]^{2}+4 a_{1} \beta\left(b \beta+c_{1} \delta\right)}}{2\left(b \beta+c_{1} \delta\right)}, \quad y_{*}=\frac{\delta}{\beta} x_{*} .
$$

The Jacobian matrix of system (1.4) at $E_{1}$ takes the form

$$
J\left(E_{1}\right)=\left(\begin{array}{cc}
-e^{-\lambda \tau_{1}} & -\frac{1}{a_{1}+b} \\
0 & \delta
\end{array}\right)
$$

The characteristic equation of system (1.4) at $E_{1}$ is of the form

$$
(\lambda-\delta)\left(\lambda+e^{-\lambda \tau_{1}}\right)=0
$$

Clearly, the boundary equilibrium $E_{1}(1,0)$ is unable. 
Next, we discuss the existence of Hopf bifurcation at the positive equilibrium $E\left(x_{*}, y_{*}\right)$. Let $x(t)=z_{1}(t)+x_{*}, y(t)=z_{2}(t)+y_{*}$, and still denote $z_{1}(t)$ and $z_{2}(t)$ by $x(t)$ and $y(t)$, respectively, then system (1.4) becomes

$$
\begin{aligned}
& \frac{d x}{d t}=a_{11} x(t)+a_{12} y(t)+b_{11} x\left(t-\tau_{1}\right)+\sum_{i+j+k \geq 2} f_{1}^{i j k} x^{i} y^{j} x^{k}\left(t-\tau_{1}\right), \\
& \frac{d y}{d t}=c_{21} x\left(t-\tau_{2}\right)+c_{22} y\left(t-\tau_{2}\right)+\sum_{i+j+k \geq 2} f_{2}^{i j k} y^{i} x^{j}\left(t-\tau_{2}\right) y^{k}\left(t-\tau_{2}\right),
\end{aligned}
$$

where

$$
\begin{gathered}
a_{11}=\frac{b x_{*} y_{*}}{\left(a_{1}+b x_{*}+c_{1} y_{*}\right)^{2}}, \quad a_{12}=-\frac{\left(a_{1}+b x_{*}\right) x_{*}}{\left(a_{1}+b x_{*}+c_{1} y_{*}\right)^{2}}, \\
b_{11}=-x_{* \prime} \quad c_{21}=\frac{\delta^{2}}{\beta}, \quad c_{22}=-\delta \\
f_{1}=x\left(1-x\left(t-\tau_{1}\right)\right)-\frac{x y}{a_{1}+b x+c_{1} y^{\prime}}, \quad f_{2}=y\left(\delta-\beta \frac{y\left(t-\tau_{2}\right)}{x\left(t-\tau_{2}\right)}\right), \\
f_{1}^{i j k} x^{i} y^{j} x^{k}\left(t-\tau_{1}\right)=\left.\frac{1}{i ! j ! k !} \frac{\partial^{i+j+k} f_{1}}{\partial x^{i} \partial y^{j} \partial x^{k}\left(t-\tau_{1}\right)}\right|_{\left(x_{*}, y_{*}\right)} \\
f_{2}^{i j k} y^{i} x^{j}\left(t-\tau_{2}\right) y^{k}\left(t-\tau_{2}\right)=\left.\frac{1}{i ! j ! k !} \frac{\partial^{i+j+k} f_{2}}{\partial y^{i} \partial x^{j}\left(t-\tau_{2}\right) \partial y^{k}\left(t-\tau_{2}\right)}\right|_{\left(x_{*}, y_{*}\right)} .
\end{gathered}
$$

Then we can obtain the linearized system of system (1.4)

$$
\begin{gathered}
\frac{d x}{d t}=a_{11} x(t)+a_{12} y(t)+b_{11} x\left(t-\tau_{1}\right), \\
\frac{d y}{d t}=c_{21} x\left(t-\tau_{2}\right)+c_{22} y\left(t-\tau_{2}\right) .
\end{gathered}
$$

The characteristic equation of system (2.6) is

$$
\lambda^{2}-a_{11} \lambda-b_{11} \lambda e^{-\lambda \tau_{1}}+\left(a_{11} c_{22}-a_{12} c_{21}-c_{22} \lambda\right) e^{-\lambda \tau_{2}}+b_{11} c_{22} e^{-\lambda\left(\tau_{1}+\tau_{2}\right)}=0 .
$$

Case 1. $\tau_{1}=\tau_{2}=\tau=0$.

The characteristic equation of system (1.4) becomes

$$
\lambda^{2}+(A+B+D) \lambda+C+E=0,
$$

where

$$
A=-a_{11}, \quad B=-b_{11}, \quad C=a_{11} c_{22}-a_{12} c_{21}, \quad D=-c_{22}, \quad E=b_{11} c_{22} .
$$


It is easy to verify that

$$
C+E=\delta x_{*}+\frac{a_{1} \delta^{2} x_{*}}{\beta\left(a_{1}+b x_{*}+c_{1} y_{*}\right)^{2}}>0
$$

Therefore, if $\left(H_{1}\right): A+B+D>0$, the roots of (2.8) must have negative real parts. Then, we know that the positive equilibrium $E_{*}\left(x_{*}, y_{*}\right)$ of system (1.4) is locally stable in the absence of delay, if $\left(H_{1}\right)$ holds.

Case 2. $\tau_{1}=\tau_{2}=\tau>0$.

The associated characteristic equation of the system is

$$
\lambda^{2}+A_{1} \lambda+\left(B_{1}+C_{1} \lambda\right) e^{-\lambda \tau}+D_{1} e^{-2 \lambda \tau}=0
$$

where

$$
A_{1}=-a_{11}, \quad B_{1}=a_{11} c_{22}-a_{12} c_{21}, \quad C_{1}=-\left(b_{11}+c_{22}\right), \quad D_{1}=b_{11} c_{22} .
$$

Multiplying $e^{\lambda \tau}$ on both sides of (2.11), we can obtain

$$
\left(\lambda^{2}+A_{1} \lambda\right) e^{\lambda \tau}+\left(B_{1}+C_{1} \lambda\right)+D_{1} e^{-\lambda \tau}=0
$$

Now, for $\tau>0$, if $\lambda=i \omega(\omega>0)$ be a root of (2.13). Then, we have

$$
\begin{aligned}
& \left(D_{1}-\omega^{2}\right) \cos \tau \omega-A_{1} \omega \sin \tau \omega=-B_{1} \\
& \left(D_{1}+\omega^{2}\right) \sin \tau \omega-A_{1} \omega \cos \tau \omega=C_{1} \omega .
\end{aligned}
$$

It follows from (2.14) that

$$
\sin \tau \omega=\frac{C_{1} \omega^{2}+\left(A_{1} B_{1}-C_{1} D_{1}\right) \omega}{\omega^{4}+A_{1}^{2} \omega^{2}-D_{1}^{2}}, \quad \cos \tau \omega=\frac{\left(B_{1}-A_{1} C_{1}\right) \omega^{2}+B_{1} D_{1}}{\omega^{4}+A_{1}^{2} \omega^{2}-D_{1}^{2}}
$$

Then we have

$$
\omega^{8}+e_{3} \omega^{6}+e_{2} \omega^{4}+e_{1} \omega^{2}+e_{0}=0
$$

where

$$
\begin{gathered}
e_{3}=2 A_{1}^{2}-C_{1}^{2}, \quad e_{2}=A_{1}^{4}+2 C_{1}^{2} D_{1}-A_{1}^{2} C_{1}^{2}-B_{1}^{2}-2 D_{1}^{2}, \\
e_{1}=4 A_{1} B_{1} C_{1} D_{1}-A_{1}^{2} B_{1}^{2}-C_{1}^{2} D_{1}^{2}-2 A_{1}^{2} D_{1}^{2}-2 B_{1}^{2} D_{1}, \quad e_{0}=D_{1}^{4}-B_{1}^{2} D_{1}^{2} .
\end{gathered}
$$


Let $v=\omega^{2}$, then $(2.16)$ becomes

$$
v^{4}+e_{3} v^{3}+e_{2} v^{2}+e_{1} v+e_{0}=0
$$

Next, we give the following assumption. $\left(H_{2}\right)$ : (2.18) has at least one positive real root.

Suppose that $\left(\mathrm{H}_{2}\right)$ holds. Without loss of generality, we assume that (2.18) has four real positive roots, which are defined by $v_{1}, v_{2}, v_{3}$, and $v_{4}$, respectively. Then (2.16) has four positive roots $\omega_{k}=\sqrt{v_{k}}, k=1,2,3,4$. Therefore,

$$
\tau_{k}^{(j)}=\frac{1}{\omega_{k}} \arccos \left(\frac{\left(B_{1}-A_{1} C_{1}\right) \omega_{k}^{2}+B_{1} D_{1}}{\omega_{k}^{4}+A_{1}^{2} \omega_{k}^{2}-D_{1}^{2}}+2 j \pi\right), \quad k=1,2,3,4 ; j=0,1,2 \ldots
$$

Then we can know that $\pm i \omega_{k}$ are a pair of purely imaginary roots of (2.11) with $\tau=\tau_{k}^{(j)}$. Define

$$
\tau_{0}=\tau_{k}^{(0)}=\min \left\{\tau_{k}^{(0)}\right\}, \quad \omega_{0}=\omega_{k_{0}}, \quad k=1,2,3,4
$$

Let $\lambda(\tau)=\alpha(\tau)+i \omega(\tau)$ be the root of (2.11) near $\tau=\tau_{0}$ which satisfies $\alpha\left(\tau_{0}\right)=0, \omega\left(\tau_{0}\right)=\omega_{0}$. Taking the derivative of $\lambda$ with respect to $\tau$ in (2.13), we obtain

$$
C_{1} \frac{d \lambda}{d \tau}+\left(2 \lambda+A_{1}\right) e^{\lambda \tau} \frac{d \lambda}{d \tau}+\left(\lambda^{2}+A_{1} \lambda\right) e^{\lambda \tau}\left(\lambda+\tau \frac{d \lambda}{d \tau}\right)-D_{1} e^{-\lambda \tau}\left(\lambda+\tau \frac{d \lambda}{d \tau}\right)=0
$$

it follows that

$$
\frac{d \lambda}{d \tau}=\frac{\lambda\left(D_{1} e^{-\lambda \tau}-\left(\lambda^{2}+A_{1} \lambda\right) e^{\lambda \tau}\right)}{\left(2 \lambda+A_{1}\right) e^{\lambda \tau}+C_{1}-\tau\left(D_{1} e^{-\lambda \tau}-\left(\lambda^{2}+A_{1} \lambda\right) e^{\lambda \tau}\right)} .
$$

Thus

$$
\left[\frac{d \lambda}{d \tau}\right]^{-1}=\frac{\left(2 \lambda+A_{1}\right) e^{\lambda \tau}+C_{1}}{D_{1} \lambda e^{-\lambda \tau}-\left(\lambda^{3}+A_{1} \lambda^{2}\right) e^{\lambda \tau}}-\frac{\tau}{\lambda} .
$$

Let

$$
\begin{gathered}
\Lambda_{1}=\left(D_{1} \omega_{0}-\omega_{0}^{3}\right) \sin \tau_{0} \omega_{0}+A_{1} \omega_{0}^{2} \cos \tau_{0} \omega_{0}, \quad \Lambda_{2}=\left(D_{1} \omega_{0}+\omega_{0}^{3}\right) \cos \tau_{0} \omega_{0}+A_{1} \omega_{0}^{2} \sin \tau_{0} \omega_{0} \\
\Lambda_{3}=A_{1} \cos \tau_{0} \omega_{0}-2 \omega_{0} \sin \tau_{0} \omega_{0}+C_{1}, \quad \Lambda_{4}=A_{1} \sin \tau_{0} \omega_{0}-2 \omega_{0} \cos \tau_{0} \omega_{0} .
\end{gathered}
$$

Substitute $\lambda=i \omega_{0}\left(\omega_{0}>0\right)$ into (2.23), we can get

$$
\operatorname{Re}\left[\frac{d \lambda\left(\tau_{0}\right)}{d \tau}\right]^{-1}=\operatorname{Re}\left[\frac{\left(2 \lambda+A_{1}\right) e^{\lambda \tau}+C_{1}}{D_{1} \lambda e^{-\lambda \tau}-\left(\lambda^{3}+A_{1} \lambda^{2}\right) e^{\lambda \tau}}\right]_{\lambda=i \omega_{0}}=\frac{\Lambda_{1} \times \Lambda_{3}+\Lambda_{2} \times \Lambda_{4}}{\Lambda_{1}^{2}+\Lambda_{2}^{2}}
$$


Noting that

$$
\operatorname{signRe}\left[\frac{d \lambda\left(\tau_{0}\right)}{d \tau}\right]=\operatorname{sign}\left[\frac{d \operatorname{Re} \lambda\left(\tau_{0}\right)}{d \tau}\right]^{-1}
$$

Therefore, we make the following assumption in order to give the main results: $\left(H_{3}\right): \Lambda_{1} \times$ $\Lambda_{3}+\Lambda_{2} \times \Lambda_{4} \neq 0$. Then, by Corollary 2.4 in $[23,24]$, we have the following theorem.

Theorem 2.1. For system (1.4), if the conditions $\left(H_{1}\right)-\left(H_{3}\right)$ hold, then the equilibrium $E_{*}\left(x_{*}, y_{*}\right)$ of system (1.4) is asymptotically stable for $\tau \in\left[0, \tau_{0}\right)$ and unstable when $\tau>\tau_{0}$. And system (1.4) has a branch of periodic solution bifurcation from the zero solution near $\tau=\tau_{0}$.

Case 3. $\tau_{1} \neq \tau_{2}, \tau_{1}>0$ and $\tau_{2}>0$.

The associated characteristic equation of the system is

$$
\lambda^{2}+A_{2} \lambda+B_{2} \lambda e^{-\lambda \tau_{1}}+\left(C_{2}+D_{2} \lambda\right) e^{-\lambda \tau_{2}}+E_{2} e^{-\lambda\left(\tau_{1}+\tau_{2}\right)}
$$

where

$$
A_{2}=-a_{11}, \quad B_{2}=-b_{11}, \quad C_{2}=a_{11} c_{22}-a_{12} c_{21}, \quad D_{2}=-c_{22}, \quad E_{2}=b_{11} c_{22} .
$$

We consider (2.27) with $\tau_{2}$ in its stable interval, regarding $\tau_{1}$ as a parameter. Without loss of generality, we consider system (1.4) under the case considered in [16], and $\tau_{2} \in\left[0, \tau_{20}\right) . \tau_{20}$ is defined as in [16] and can be obtained by

$$
\tau_{20}=\frac{1}{\omega_{+}} \arccos \left[\frac{\left(a_{11} c_{22}+b_{11} c_{22}-a_{12} c_{21}\right) \omega_{+}^{2}-\left(a_{11}+b_{11}\right) c_{22} \omega_{+}^{2}}{\left(a_{11} c_{22}+b_{11} c_{22}-a_{12} c_{21}\right)^{2}+\left(c_{22} \omega_{+}\right)^{2}}\right],
$$

with

$$
\omega_{+}=\sqrt{\frac{-\left(\left(a_{11}+b_{11}\right)^{2}-c_{22}^{2}\right)+\sqrt{\left(\left(a_{11}+b_{11}\right)^{2}-c_{22}^{2}\right)^{2}+4\left(a_{11} c_{22}+b_{11} c_{22}-a_{12} c_{21}\right)^{2}}}{2}} .
$$

Let $\lambda=i \omega(\omega>0)$ be a root of (2.27). Then we obtain

$$
\begin{gathered}
\left(B_{2} \omega-E_{2} \sin \tau_{2} \omega\right) \sin \tau_{1} \omega+E_{2} \cos \tau_{2} \omega \cos \tau_{1} \omega=\omega^{2}-C_{2} \cos \tau_{2} \omega-D_{2} \omega \sin \tau_{2} \omega, \\
\left(B_{2} \omega-E_{2} \sin \tau_{2} \omega\right) \cos \tau_{1} \omega-E_{2} \cos \tau_{2} \omega \sin \tau_{1} \omega=C_{2} \sin \tau_{2} \omega-D_{2} \omega \cos \tau_{2} \omega-A_{2} \omega .
\end{gathered}
$$

It follows from (2.31) that

$$
\sin \tau_{1} \omega=\frac{M_{1} N_{1}-M_{2} N_{2}}{M_{1}^{2}+M_{2}^{2}}, \quad \cos \tau_{1} \omega=\frac{M_{1} N_{2}+M_{2} N_{1}}{M_{1}^{2}+M_{2}^{2}},
$$


With

$$
\begin{gathered}
M_{1}=B_{2} \omega-E_{2} \sin \tau_{2} \omega, \quad M_{2}=E_{2} \cos \tau_{2} \omega, \\
N_{1}=\omega^{2}-C_{2} \cos \tau_{2} \omega-D_{2} \omega \sin \tau_{2} \omega, \quad N_{2}=-A_{2} \omega+C_{2} \sin \tau_{2} \omega-D_{2} \omega \cos \tau_{2} \omega .
\end{gathered}
$$

Then we have

$$
P_{1}(\omega)+P_{2}(\omega) \sin \tau_{2} \omega+P_{3}(\omega) \cos \tau_{2} \omega=0
$$

where

$$
\begin{gathered}
P_{1}(\omega)=\omega^{4}+\left(A_{2}^{2}+D_{2}^{2}-B_{2}^{2}\right) \omega^{2}+C_{2}^{2}-E_{2}^{2} \\
P_{2}(\omega)=-2 D_{2} \omega^{3}-2 A_{2} C_{2} \omega+2 B_{2} E_{2} \omega, \quad P_{3}(\omega)=2\left(A_{2} D_{2}-C_{2}\right) \omega^{2} .
\end{gathered}
$$

Suppose that $\left(H_{4}\right):(2.34)$ has at least finite positive roots. If $\left(H_{4}\right)$ holds, we define the roots of $(2.34)$ as $\omega_{1}, \omega_{2}, \ldots, \omega_{k}$. Then, for every fixed $\omega_{i}(i=1,2, \ldots, k)$, there exists a sequence $\left\{\tau_{1_{i}}^{(j)} \mid j=1,2, \ldots\right\}$ which satisfies (2.34). Let

$$
\tau_{1 *}=\min \left\{\tau_{1_{i}}^{(j)} \mid i=1,2, \ldots, k, j=0,1, \ldots\right\}
$$

When $\tau_{1}=\tau_{1 *},(2.27)$ has a pair of purely imaginary roots $\pm i \omega_{*}$ for $\tau_{2} \in\left[0, \tau_{20}\right)$.

To verify the transversality condition of Hopf bifurcation, we take the derivative of $\lambda$ with respect to $\tau_{1}$ in (2.27), we can obtain

$$
\frac{d \lambda}{d \tau_{1}}=\frac{\lambda e^{-\lambda \tau_{1}}\left(B_{2} \lambda+E_{2} e^{-\lambda \tau_{2}}\right)}{\left(2 \lambda+A_{2}+B_{2} e^{-\lambda \tau_{1}}+D_{2} e^{-\lambda \tau_{2}}\right)-\tau_{1} e^{-\lambda \tau_{1}}\left(B_{2} \lambda+E_{2} e^{-\lambda \tau_{2}}\right)-\tau_{2} e^{-\lambda \tau_{2}}\left(C_{2}+D_{2} \lambda+E_{2} e^{-\lambda \tau_{1}}\right)}
$$

Thus

$$
\left[\frac{d \lambda}{d \tau_{1}}\right]^{-1}=\frac{2 \lambda+A_{2}+B_{2} e^{-\lambda \tau_{1}}+D_{2} e^{-\lambda \tau_{2}}-\tau_{2} e^{-\lambda \tau_{2}}\left(C_{2}+D_{2} \lambda+E_{2} e^{-\lambda \tau_{1}}\right)}{\lambda e^{-\lambda \tau_{1}}\left(B_{2} \lambda+E_{2} e^{-\lambda \tau_{2}}\right)}-\frac{\tau_{1}}{\lambda}
$$

Substitute $\lambda=i \omega_{*}\left(\omega_{*}>0\right)$ into (2.38), we can get

$$
\operatorname{Re}\left[\frac{d \lambda\left(\tau_{1 *}\right)}{d \tau_{1}}\right]^{-1}=\frac{\Delta_{1} \times \Delta_{3}+\Delta_{2} \times \Delta_{4}}{\Delta_{1}^{2}+\Delta_{2}^{2}}
$$


where

$$
\begin{gathered}
\Delta_{1}=E_{2} \omega_{*} \cos \tau_{2} \omega_{*} \sin \tau_{1 *} \omega_{*}-\omega_{*} \cos \tau_{1 *} \omega_{*}\left(B_{2} \omega_{*}-E_{2} \omega_{*} \sin \tau_{2} \omega_{*}\right), \\
\Delta_{2}=E_{2} \omega_{*} \cos \tau_{2} \omega_{*} \cos \tau_{1 *} \omega_{*}+\omega_{*} \sin \tau_{1 *} \omega_{*}\left(B_{2} \omega_{*}-E_{2} \omega_{*} \sin \tau_{2} \omega_{*}\right), \\
\Delta_{3}=A_{2}+\left(D_{2}-\tau_{2} C_{2}\right) \cos \tau_{2} \omega_{*}-\tau_{2} D_{2} \omega_{*} \sin \tau_{2} \omega_{*} \\
\quad+\tau_{2} E_{2} \sin \tau_{2} \omega_{*} \sin \tau_{1 *} \omega_{*}+\left(B_{2}-\tau_{2} E_{2} \cos \tau_{2} \omega_{*}\right) \cos \tau_{1 *} \omega_{* \prime} \\
\Delta_{4}=2 \omega_{*}+\left(\tau_{2} C_{2}+\tau_{2} D_{2} \omega_{*}-D_{2}\right) \sin \tau_{2} \omega_{*} \\
\quad+\tau_{2} E_{2} \sin \tau_{2} \omega_{*} \cos \tau_{1 *} \omega_{*}-\left(B_{2}-\tau_{2} E_{2} \cos \tau_{2} \omega_{*}\right) \sin \tau_{1 *} \omega_{*} .
\end{gathered}
$$

Next, we make the following assumption: $\left(H_{5}\right): \Delta_{1} \times \Delta_{3}+\Delta_{2} \times \Delta_{4} \neq 0$.

Thus, by the discussion above and by the general Hopf bifurcation theorem for FDEs in Hale [25], we have the following results.

Theorem 2.2. For $\tau_{2} \in\left(0, \tau_{20}\right), \tau_{20}$ is defined by (2.29). If the conditions $\left(H_{4}\right)-\left(H_{5}\right)$ hold, then the equilibrium $E_{*}\left(x_{*}, y_{*}\right)$ of system (1.4) is asymptotically stable for $\tau_{1} \in\left[0, \tau_{1 *}\right)$ and unstable when $\tau>\tau_{1 *}$. System (1.4) has a branch of periodic solution bifurcation from the zero solution near $\tau=\tau_{1 *}$.

\section{Direction and Stability of Bifurcated Periodic Solutions}

In this section, we shall investigate the direction of the Hopf bifurcation and the stability of bifurcating periodic solution of system (1.4) w.r. to $\tau_{1}$ for $\tau_{2} \in\left(0, \tau_{20}\right)$, and $\tau_{20}$ is defined by (2.29). The idea employed here is the normal form and center manifold theory described in Hassard et al. [26]. Throughout this section, it is considered that system (1.4) undergoes the Hopf bifurcation at $\tau_{1}=\tau_{1 *}, \tau_{2} \in\left(0, \tau_{20}\right)$ at $E_{*}\left(x_{*}, y_{*}\right)$. Let $\tau_{1}=\tau_{1 *}+\mu, \mu \in R$ so that the Hopf bifurcation occurs at $\mu=0$. Without loss of generality, we assume that $\tau_{2 *}<\tau_{1 *}$, where $\tau_{2 *} \in\left(0, \tau_{20}\right)$.

Let $u_{1}(t)=x(t)-x_{*}, u_{2}(t)=y(t)-y_{*}$, and rescaling the time delay $t \rightarrow\left(t / \tau_{1}\right)$, Then system (1.4) can be transformed into an FDE in $C=C\left([-1,0], R^{2}\right)$ as:

$$
\dot{u}(t)=L_{\mu} u_{t}+F\left(\mu, u_{t}\right)
$$

where $u(t)=\left(u_{1}(t), u_{2}(t)\right)^{T} \in R^{2}$ and $L_{\mu}: C \rightarrow R^{2}, F: R \times C \rightarrow R^{2}$ are given, respectively, by

$$
\begin{gathered}
L_{\mu} \phi=\left(\tau_{1 *}+\mu\right)\left(A^{\prime} \phi(0)+C^{\prime} \phi\left(-\frac{\tau_{2 *}}{\tau_{1 *}}\right)+B^{\prime} \phi(-1)\right), \\
F(\mu, \phi)=\left(\tau_{1 *}+\mu\right)\left(f_{1}, f_{2}\right)^{T}
\end{gathered}
$$


with

$$
\begin{aligned}
A^{\prime}= & \left(\begin{array}{cc}
a_{11} & a_{12} \\
0 & 0
\end{array}\right), \quad B^{\prime}=\left(\begin{array}{cc}
b_{11} & 0 \\
0 & 0
\end{array}\right), \quad C^{\prime}=\left(\begin{array}{cc}
0 & 0 \\
c_{21} & c_{22}
\end{array}\right), \\
f_{1}= & g_{1} \phi_{1}^{2}(0)+g_{2} \phi_{1}(0) \phi_{2}(0)+g_{3} \phi_{2}^{2}(0)+g_{4} \phi_{1}(0) \phi_{1}(-1) \\
& +h_{1} \phi_{1}^{3}(0)+h_{2} \phi_{1}^{2}(0) \phi_{2}(0)+h_{3} \phi_{1}(0) \phi_{2}^{2}(0)+h_{4} \phi_{2}^{3}(0)+\cdots, \\
f_{2}= & g_{1}^{\prime} \phi_{1}^{2}\left(-\frac{\tau_{2 *}}{\tau_{1 *}}\right)+g_{2}^{\prime} \phi_{1}\left(-\frac{\tau_{2 *}}{\tau_{1 *}}\right) \phi_{2}(0)+g_{3}^{\prime} \phi_{1}\left(-\frac{\tau_{2 *}}{\tau_{1 *}}\right) \phi_{2}\left(-\frac{\tau_{2 *}}{\tau_{1 *}}\right), \\
& +g_{4}^{\prime} \phi_{2}(0) \phi_{2}\left(-\frac{\tau_{2 *}}{\tau_{1 *}}\right)+h_{1}^{\prime} \phi_{1}^{3}\left(-\frac{\tau_{2 *}}{\tau_{1 *}}\right)+h_{2}^{\prime} \phi_{1}^{2}\left(-\frac{\tau_{2 *}}{\tau_{1 *}}\right) \phi_{2}(0), \\
& +h_{3}^{\prime} \phi_{1}^{2}\left(-\frac{\tau_{2 *}}{\tau_{1 *}}\right) \phi_{2}\left(-\frac{\tau_{2 *}}{\tau_{1 *}}\right)+h_{4} \phi_{2}^{3}(0)+\cdots,
\end{aligned}
$$

where

$$
\begin{gathered}
g_{1}=\frac{b y_{*}\left(a_{1}+c_{1} y_{*}\right)}{\left(a_{1}+b x_{*}+c_{1} y_{*}\right)^{3}}, \quad g_{2}=-\frac{a_{1}^{2}+a_{1} b x_{*}+a_{1} c_{1} y_{*}+2 b c_{1} x_{*} y_{*}}{\left(a_{1}+b x_{*}+c_{1} y_{*}\right)^{3}}, \\
g_{3}=\frac{c_{1} x_{*}\left(a_{1}+b x_{*}\right)}{\left(a_{1}+b x_{*}+c_{1} y_{*}\right)^{3}}, \quad g_{4}=-1, \\
h_{1}=-\frac{b^{2} y_{*}\left(a_{1}+c_{1} y_{*}\right)}{\left(a_{1}+b x_{*}+c_{1} y_{*}\right)^{4}}, \quad h_{2}=\frac{a_{1}^{2} b+a_{1} b^{2} x_{*}+2 b^{2} c_{1} x_{*} y_{*}-b c_{1}^{2} y_{*}^{2}}{\left(a_{1}+b x_{*}+c_{1} y_{*}\right)^{4}}, \\
h_{3}=\frac{a_{1}^{2} c_{1}+a_{1} c_{1}^{2} y_{*}+2 b c_{1}^{2} x_{*} y_{*}-b^{2} c_{1} x_{*}^{2}}{\left(a_{1}+b x_{*}+c_{1} y_{*}\right)^{4}}, \quad h_{4}=-\frac{c_{1}^{2} x_{*}\left(a_{1}+b x_{*}\right)}{\left(a_{1}+b x_{*}+c_{1} y_{*}\right)^{4}}, \\
g_{1}^{\prime}=-\frac{\beta y_{*}^{2}}{x_{*}^{3}}, \quad g_{2}^{\prime}=\frac{\beta y_{*}}{x_{*}^{2}}, \quad g_{3}^{\prime}=\frac{\beta y_{*}}{x_{*}^{2}}, \quad g_{4}^{\prime}=-\frac{\beta}{x_{*}}, \\
h_{1}^{\prime}=\frac{\beta y_{*}^{2}}{x_{*}^{4}}, \quad h_{2}^{\prime}=-\frac{\beta y_{*}}{x_{*}^{3}}, \quad h_{3}^{\prime}=-\frac{\beta y_{*}}{x_{*}^{3}} .
\end{gathered}
$$

Using Riesz representation theorem, there exists a $2 \times 2$ matrix function $\eta(\theta, \mu), \theta \in[-1,0]$ whose elements are of bounded variation, such that

$$
L_{\mu} \phi=\int_{-1}^{0} d \eta(\theta, \mu) \phi(\theta), \quad \phi \in C\left([-1,0], R_{2}\right) .
$$


In fact, choosing

$$
\eta(\theta, \mu)= \begin{cases}\left(\tau_{1 *+\mu}\right)\left(A^{\prime}+C^{\prime}+B^{\prime}\right), & \theta=0, \\ \left(\tau_{1 *+\mu}\right)\left(C^{\prime}+B^{\prime}\right), & \theta \in\left[-\frac{\tau_{2 *}}{\tau_{1}}, 0\right), \\ \left(\tau_{1 *+\mu}\right) B^{\prime}, & \theta \in\left(-1,-\frac{\tau_{2 *}}{\tau_{1}}\right), \\ 0, & \theta=-1 .\end{cases}
$$

For $\phi \in C([-1,0])$, we define

$$
\begin{gathered}
A(\mu) \phi= \begin{cases}\frac{d \phi(\theta)}{d \theta}, & -1 \leq \theta<0, \\
\int_{-1}^{0} d \eta(\theta, \mu) \phi(\theta), & \theta=0\end{cases} \\
R(\mu) \phi= \begin{cases}0, & -1 \leq \theta<0 \\
F(\mu, \phi), & \theta=0 .\end{cases}
\end{gathered}
$$

Then system (3.1) can be transformed into the following operator equation

$$
\dot{u}(t)=A(\mu) u_{t}+R(\mu) u_{t}
$$

where $u_{t}=u(t+\theta)=\left(u_{1}(t+\theta), u_{2}(t+\theta)\right)$.

For $\varphi \in C^{1}\left([0,1],\left(R^{2}\right)^{*}\right)$, where $\left(R^{2}\right)^{*}$ is the 2-dimensional space of row vectors, we further define the adjoint operator $A^{*}$ of $\mathrm{A}(0)$ :

$$
A^{*}(\varphi)= \begin{cases}-\frac{d \varphi(s)}{d s}, & 0<s \leq 1, \\ \int_{-1}^{0} \varphi(-\xi) d \eta(\xi, 0), & s=0,\end{cases}
$$

and a bilinear inner product:

$$
\langle\varphi(s), \phi(\theta)\rangle=\bar{\varphi}^{T}(0) \phi(0)-\int_{\theta=-1}^{0} \int_{\xi=0}^{\theta} \bar{\varphi}^{T}(\xi-\theta) d \eta(\theta) \phi(\xi) d \xi,
$$

where $\eta(\theta)=\eta(\theta, 0)$. 
Since $\pm i \omega_{*} \tau_{1 *}$ are eigenvalues of $A(0)$, they are also eigenvalues of $A^{*}$. Let $q(\theta)=\left(1, q_{2}\right)^{T} e^{i \omega_{*} \tau_{1 *} \theta}$ be the eigenvectors of $A(0)$ corresponding to $i \omega_{*} \tau_{1 *}$ and $q^{*}(s)=$ $(1 / \rho)\left(1, q_{2}^{*}\right)^{T} e^{i \omega_{*} \tau_{1 *} s}$ be the eigenvectors of $A^{*}$ corresponding to $-i \omega_{*} \tau_{1 *}$. By a simple computation, we can get

$$
\begin{gathered}
q_{2}=\frac{i \omega_{*}-a_{11}-b_{11} e^{i \omega_{*} \tau_{1 *}}}{a_{12}}, \quad q_{2}^{*}=-\frac{i \omega_{*}+a_{11}+b_{11} e^{i \omega_{*} \tau_{1 *}}}{c_{21} e^{i \omega_{*} \tau_{2 *}}} \\
\bar{\rho}=1+q_{2} \bar{q}_{2}^{*}+b_{11} \tau_{1 *} e^{-i \omega_{*} \tau_{1 *}}+c_{21} \tau_{2 *} \bar{q}_{2}^{*} e^{-i \omega_{*} \tau_{2 *}}+c_{22} \tau_{2 *} q_{2} \bar{q}_{2}^{*} e^{-i \omega_{*} \tau_{2 *}}
\end{gathered}
$$

Then $\left\langle q^{*}, q\right\rangle=1,\left\langle q^{*}, \bar{q}\right\rangle=0$.

In the remainder of this section, Following the algorithms given in [26] and using similar computation process to that in [16], we can get that the coefficients which will be used to determine the important qualities of the bifurcating periodic solutions,

$$
\begin{aligned}
& g_{20}=\frac{2 \tau_{1 *}}{\bar{\rho}}\left\{g_{1}+g_{2} q^{(2)}(0)+g_{3}\left(q^{(2)}(0)\right)^{2}+g_{4} q^{(1)}(-1)\right. \\
& +\bar{q}_{2}^{*}\left(g_{1}^{\prime}\left(q^{(1)}\left(-\frac{\tau_{2 *}}{\tau_{1 *}}\right)\right)^{2}+g_{2}^{\prime} q^{(1)}\left(-\frac{\tau_{2 *}}{\tau_{1^{*}}}\right) q^{(2)}(0)+g_{3}^{\prime} q^{(1)}\left(-\frac{\tau_{2 *}}{\tau_{1 *}}\right) q^{(2)}\left(-\frac{\tau_{2 *}}{\tau_{1 *}}\right)\right. \\
& \left.\left.+g_{4}^{\prime} q^{(2)}(0) q^{(2)}\left(-\frac{\tau_{2 *}}{\tau_{1 *}}\right)\right)\right\}, \\
& g_{11}=\frac{\tau_{1 *}}{\bar{\rho}}\left\{2 g_{1}+g_{2}\left(q^{(2)}(0)+\bar{q}^{(2)}(0)\right)+2 g_{3} q^{(2)}(0) \bar{q}^{(2)}(0)+g_{4}\left(q^{(1)}(-1)+\bar{q}^{(1)}(-1)\right)\right. \\
& +\bar{q}_{2}^{*}\left(2 g_{1}^{\prime} q^{(1)}\left(-\frac{\tau_{2 *}}{\tau_{1 *}}\right) \bar{q}^{(1)}\left(-\frac{\tau_{2 *}}{\tau_{1 *}}\right)+g_{2}^{\prime}\left(q^{(1)}\left(-\frac{\tau_{2 *}}{\tau_{1 *}}\right) \bar{q}^{(2)}(0)+\bar{q}^{(1)}\left(-\frac{\tau_{2 *}}{\tau_{1 *}}\right) q^{(2)}(0)\right)\right. \\
& +g_{3}^{\prime}\left(q^{(1)}\left(-\frac{\tau_{2 *}}{\tau_{1 *}}\right) \bar{q}^{(2)}\left(-\frac{\tau_{2 *}}{\tau_{1 *}}\right)+\bar{q}^{(1)}\left(-\frac{\tau_{2 *}}{\tau_{1 *}}\right) q^{(2)}\left(-\frac{\tau_{2 *}}{\tau_{1 *}}\right)\right) \\
& \left.\left.+g_{4}^{\prime}\left(q^{(2)}(0) \bar{q}^{(2)}\left(-\frac{\tau_{2 *}}{\tau_{1 *}}\right)+\bar{q}^{(2)}(0) q^{(2)}\left(-\frac{\tau_{2 *}}{\tau_{1 *}}\right)\right)\right)\right\}, \\
& g_{02}=\frac{2 \tau_{1 *}}{\bar{\rho}}\left\{g_{1}+g_{2} \bar{q}^{(2)}(0)+g_{3}\left(\bar{q}^{(2)}(0)\right)^{2}+g_{4} \bar{q}^{(1)}(-1)\right. \\
& +\bar{q}_{2}^{*}\left(g_{1}^{\prime}\left(\bar{q}^{(1)}\left(-\frac{\tau_{2 *}}{\tau_{1 *}}\right)\right)^{2}+g_{2}^{\prime} \bar{q}^{(1)}\left(-\frac{\tau_{2 *}}{\tau_{1 *}}\right) \bar{q}^{(2)}(0)+g_{3}^{\prime} \bar{q}^{(1)}\left(-\frac{\tau_{2 *}}{\tau_{1 *}}\right) \bar{q}^{(2)}\left(-\frac{\tau_{2 *}}{\tau_{1 *}}\right)\right. \\
& \left.\left.+g_{4}^{\prime} \bar{q}^{(2)}(0) \bar{q}^{(2)}\left(-\frac{\tau_{2 *}}{\tau_{1 *}}\right)\right)\right\}
\end{aligned}
$$




$$
\begin{aligned}
& g_{21}=\frac{2 \tau_{1 *}}{\bar{\rho}}\left\{g_{1}\left(W_{20}^{(1)}(0)+2 W_{11}^{(1)}(0)\right)\right. \\
& +g_{2}\left(\frac{1}{2} W_{20}^{(2)}(0)+W_{11}^{(2)}(0)+\frac{1}{2} W_{20}^{(1)}(0) \bar{q}^{(2)}(0)+W_{11}^{(1)}(0) q^{(2)}(0)\right) \\
& +g_{3}\left(W_{20}^{(2)}(0) \bar{q}^{(2)}(0)+2 W_{11}^{(2)}(0) q^{(2)}(0)\right) \\
& +g_{4}\left(\frac{1}{2} W_{20}^{(1)}(-1)+W_{11}^{(1)}(-1)+\frac{1}{2} W_{20}^{(1)}(0) \bar{q}^{(1)}(-1)+W_{11}^{(1)}(0) q^{(1)}(-1)\right)+3 h_{1} \\
& +h_{2}\left(2 q^{(2)}(0)+\bar{q}^{(2)}(0)\right)+h_{3}\left(2 q^{(2)}(0) \bar{q}^{(2)}(0)+\left(q^{(2)}(0)\right)^{2}\right)+3 h_{4}\left(q^{(2)}(0)\right)^{2} \bar{q}^{(2)}(0) \\
& +\bar{q}_{2}^{*}\left(g_{1}^{\prime}\left(W_{20}^{(1)}\left(-\frac{\tau_{2 *}}{\tau_{1 *}}\right) \bar{q}^{(1)}\left(-\frac{\tau_{2 *}}{\tau_{1 *}}\right)+2 W_{11}^{(1)}\left(-\frac{\tau_{2 *}}{\tau_{1 *}}\right) q^{(1)}\left(-\frac{\tau_{2 *}}{\tau_{1 *}}\right)\right)\right. \\
& +g_{2}^{\prime}\left(\frac{1}{2} W_{20}^{(1)}\left(-\frac{\tau_{2 *}}{\tau_{1 *}}\right) \bar{q}^{(2)}(0)+W_{11}^{(1)}\left(-\frac{\tau_{2 *}}{\tau_{1 *}}\right) q^{(2)}(0)+\frac{1}{2} W_{20}^{(2)}(0) \bar{q}^{(1)}\left(-\frac{\tau_{2 *}}{\tau_{1 *}}\right)\right. \\
& \left.+W_{11}^{(2)}(0) q^{(1)}\left(-\frac{\tau_{2 *}}{\tau_{1 *}}\right)\right) \\
& +g_{3}^{\prime}\left(\frac{1}{2} W_{20}^{(1)}\left(-\frac{\tau_{2 *}}{\tau_{1 *}}\right) \bar{q}^{(2)}\left(-\frac{\tau_{2 *}}{\tau_{1 *}}\right)+W_{11}^{(1)}\left(-\frac{\tau_{2 *}}{\tau_{1 *}}\right) q^{(2)}\left(-\frac{\tau_{2 *}}{\tau_{1 *}}\right)\right. \\
& \left.+\frac{1}{2} W_{20}^{(2)}\left(-\frac{\tau_{2 *}}{\tau_{1 *}}\right) \bar{q}^{(1)}\left(-\frac{\tau_{2 *}}{\tau_{1 *}}\right)\right) \\
& +g_{4}^{\prime}\left(\frac{1}{2} W_{20}^{(2)}(0) \bar{q}^{(2)}\left(-\frac{\tau_{2 *}}{\tau_{1 *}}\right)+W_{11}^{(2)}(0) q^{(2)}\left(-\frac{\tau_{2 *}}{\tau_{1 *}}\right)+\frac{1}{2} W_{20}^{(2)}\left(-\frac{\tau_{2 *}}{\tau_{1 *}}\right) \bar{q}^{(2)}(0)\right. \\
& \left.+W_{11}^{(2)}\left(-\frac{\tau_{2 *}}{\tau_{1 *}}\right) q^{(2)}(0)\right) \\
& +3 h_{1}^{\prime}\left(q^{(1)}\left(-\frac{\tau_{2 *}}{\tau_{1 *}}\right)\right)^{2} \bar{q}^{(1)}\left(-\frac{\tau_{2 *}}{\tau_{1 *}}\right) \\
& +h_{2}^{\prime}\left(\left(q^{(1)}\left(-\frac{\tau_{2 *}}{\tau_{1 *}}\right)\right)^{2} \bar{q}^{(2)}(0)+2 q^{(1)}\left(-\frac{\tau_{2 *}}{\tau_{1 *}}\right) \bar{q}^{(1)}\left(-\frac{\tau_{2 *}}{\tau_{1 *}}\right) q^{(2)}(0)\right) \\
& \left.\left.+h_{3}^{\prime}\left(\left(q^{(1)}\left(-\frac{\tau_{2 *}}{\tau_{1 *}}\right)\right)^{2} \bar{q}^{(2)}\left(-\frac{\tau_{2 *}}{\tau_{1 *}}\right)+2 q^{(1)}\left(-\frac{\tau_{2 *}}{\tau_{1 *}}\right) \bar{q}^{(1)}\left(-\frac{\tau_{2 *}}{\tau_{1 *}}\right) q^{(2)}\left(-\frac{\tau_{2 *}}{\tau_{1 *}}\right)\right)\right)\right\},
\end{aligned}
$$

with

$$
\begin{gathered}
W_{20}(\theta)=\frac{i g_{20} q(0)}{\tau_{1 *} \omega_{*}} e^{i \tau_{1 *} \omega_{*} \theta}+\frac{i \bar{g}_{02} \bar{q}(0)}{3 \tau_{1 *} \omega_{*}} e^{-i \tau_{1 *} \omega_{*} \theta}+E_{20} e^{2 i \tau_{1 *} \omega_{*} \theta}, \\
W_{11}(\theta)=-\frac{i g_{11} q(0)}{\tau_{1 *} \omega_{*}} e^{i \tau_{1 *} \omega_{*} \theta}+\frac{i \bar{g}_{11} \bar{q}(0)}{\tau_{1 *} \omega_{*}} e^{-i \tau_{1 *} \omega_{*} \theta}+E_{11},
\end{gathered}
$$


where $E_{20}$ and $E_{11}$ can be computed as the following equations, respectively,

$$
\begin{gathered}
E_{20}=2\left(\begin{array}{c}
E_{20}^{(1)} \\
E_{20}^{(2)}
\end{array}\right) \times\left(\begin{array}{cc}
2 i \omega_{*}-a_{11}-b_{11} e^{-2 i \tau_{1 *} \omega_{*}} & -a_{12} \\
-c_{21} e^{-2 i \tau_{2 *} \omega_{*}} & 2 i \omega_{*}-c_{22} e^{-2 i \tau_{2 *} \omega_{*}}
\end{array}\right)^{-1}, \\
E_{11}=-\left(\begin{array}{c}
E_{11}^{(1)} \\
E_{11}^{(2)}
\end{array}\right) \times\left(\begin{array}{cc}
a_{11}+b_{11} & a_{12} \\
c_{21} & c_{22}
\end{array}\right)^{-1},
\end{gathered}
$$

with

$$
\begin{gathered}
E_{20}^{(1)}=g_{1}+g_{2} q^{(2)}(0)+g_{3}\left(q^{(2)}(0)\right)^{2}+g_{4} q^{(1)}(-1), \\
E_{20}^{(2)}=g_{1}^{\prime}\left(q^{(1)}\left(-\frac{\tau_{2 *}}{\tau_{1 *}}\right)\right)^{2}+g_{2}^{\prime} q^{(1)}\left(-\frac{\tau_{2 *}}{\tau_{1 *}}\right) q^{(2)}(0) \\
+g_{3}^{\prime} q^{(1)}\left(-\frac{\tau_{2 *}}{\tau_{1 *}}\right) q^{(2)}\left(-\frac{\tau_{2 *}}{\tau_{1 *}}\right)+g_{4}^{\prime} q^{(2)}(0) q^{(2)}\left(-\frac{\tau_{2 *}}{\tau_{1 *}}\right), \\
E_{11}^{(1)}=2 g_{1}+g_{2}\left(q^{(2)}(0)+\bar{q}^{(2)}(0)\right)+2 g_{3} q^{(2)}(0) \bar{q}^{(2)}(0)+g_{4}\left(q^{(1)}(-1)+\bar{q}^{(1)}(-1)\right), \\
E_{11}^{(2)}=2 g_{1}^{\prime} q^{(1)}\left(-\frac{\tau_{2 *}}{\tau_{1 *}}\right) \bar{q}^{(1)}\left(-\frac{\tau_{2 *}}{\tau_{1 *}}\right)+g_{2}^{\prime}\left(q^{(1)}\left(-\frac{\tau_{2 *}}{\tau_{1 *}}\right) \bar{q}^{(2)}(0)+\bar{q}^{(1)}\left(-\frac{\tau_{2 *}}{\tau_{1 *}}\right) q^{(2)}(0)\right) \\
+g_{3}^{\prime}\left(q^{(1)}\left(-\frac{\tau_{2 *}}{\tau_{1 *}}\right) \bar{q}^{(2)}\left(-\frac{\tau_{2 *}}{\tau_{1 *}}\right)+\bar{q}^{(1)}\left(-\frac{\tau_{2 *}}{\tau_{1 *}}\right) q^{(2)}\left(-\frac{\tau_{2 *}}{\tau_{1 *}}\right)\right) \\
+g_{4}^{\prime}\left(q^{(2)}(0) \bar{q}^{(2)}\left(-\frac{\tau_{2 *}}{\tau_{1 *}}\right)+q^{-(2)}(0) q^{(2)}\left(-\frac{\tau_{2 *}}{\tau_{1 *}}\right)\right) .
\end{gathered}
$$

Therefore, we can calculate the following values:

$$
\begin{gathered}
C_{1}(0)=\frac{i}{2 \tau_{1 *} \omega_{*}}\left(g_{11} g_{20}-2\left|g_{11}\right|^{2}-\frac{\left|g_{02}\right|^{2}}{3}\right)+\frac{g_{21}}{2}, \\
\mu_{2}=-\frac{\operatorname{Re}\left\{C_{1}(0)\right\}}{\operatorname{Re}\left\{\lambda^{\prime}\left(\tau_{1 *}\right)\right\}} \\
\beta_{2}=2 \operatorname{Re}\left\{C_{1}(0)\right\}, \\
T_{2}=-\frac{\operatorname{Im}\left\{C_{1}(0)\right\}+\mu_{2} \operatorname{Im}\left\{\lambda^{\prime}\left(\tau_{1 *}\right)\right\}}{\tau_{1 *} \omega_{*}} .
\end{gathered}
$$

Based on the discussion above, we can obtain the following results.

Theorem 3.1. The direction of the Hopf bifurcation is determined by the sign of $\mu_{2}$ : if $\mu_{2}>0\left(\mu_{2}<\right.$ 0 ), the Hopf bifurcation is supercritical (subcritical). The stability of bifurcating periodic solutions is determined by the sign of $\beta_{2}$ : if $\beta_{2}<0\left(\beta_{2}>0\right)$, the bifurcating periodic solutions are stable (unstable). 
The period of the bifurcating periodic solutions is determined by the sign of $T_{2}$ : if $T_{2}>0\left(T_{2}<0\right)$, the period of the bifurcating periodic solutions increases (decreases).

\section{Numerical Example}

In order to support the analytic results obtained above, we give some numerical simulations in this section. We only study the most important steady state, namely, the positive steady state. We consider the following system by taking the same coefficients as in [16]:

$$
\begin{gathered}
\frac{d x}{d t}=x\left(1-x\left(t-\tau_{1}\right)\right)-\frac{x y}{0.01+3 x+y}, \\
\frac{d y}{d t}=y\left[3.5-2 \frac{y\left(t-\tau_{2}\right)}{x\left(t-\tau_{2}\right)}\right]
\end{gathered}
$$

where we get the positive equilibrium $E_{*}(0.6328,1.1074)$. For system (4.1), we can get that $A+B+D=3.9017>0$, namely, the condition $\left(H_{1}\right)$ holds.

For $\tau_{1}=\tau_{2}=\tau \neq 0$. By a simple computation, we obtain that (2.18) has two positive roots: $v_{1}=12.0256, v_{2}=0.3915$. Thus, we know that the condition $\left(H_{2}\right)$ holds. Further, we get $\omega_{0}=3.4678, \tau_{0}=0.4292$. In addition, we have $\Lambda_{1} \times \Lambda_{3}+\Lambda_{2} \times \Lambda_{4}=94.4826>0$. Therefore, the condition $\left(H_{3}\right)$ is satisfied. Hence, from Theorem 2.1, we conclude that the positive equilibrium $E_{*}(0.6328,1.1074)$ is asymptotically stable when $\tau \in\left[0, \tau_{0}\right)$. The corresponding waveform and the phase plot are illustrated by Figure 1. When the delay $\tau$ passes through the critical value $\tau_{0}$ the positive equilibrium $E_{*}(0.6328,1.1074)$ will lose its stability and a Hopf bifurcation occurs, and a family of periodic solution bifurcates from the positive equilibrium $E_{*}(0.6328,1.1074)$. This property is illustrated by the numerical simulation in Figure 2.

For $\tau_{1} \neq \tau_{2}, \tau_{1}>0$ and $\tau_{2}>0$. Regard $\tau_{1}$ as a parameter and let $\tau_{2}=0.36 \in\left[0, \tau_{20}\right)$, and from (2.29) we can obtian $\tau_{20}=0.4037$. Then by a simple computation, we can obtain (2.34) has a positive root $\omega_{*}=0.6792$. Thus, the condition $\left(H_{4}\right)$ holds. Further, we we can get $\tau_{1 *}=2.3211$ and $\Delta_{1} \times \Delta_{3}+\Delta_{2} \times \Delta_{4}=3.7131>0$. Namely, the condition $\left(H_{5}\right)$ holds. Thus, by Theorem 2.2 , the positive equilibrium $E_{*}(0.6328,1.1074)$ is asymptotically stable when $\tau_{1} \in\left[0, \tau_{1 *}\right)$ and unstable when $\tau_{1}>\tau_{1 *}$, and a family of periodic solution bifurcates from the positive equilibrium $E_{*}(0.6328,1.1074)$. The corresponding waveform and the phase plot are illustrated by Figures 3 and 4, respectively. In addition, from (3.16), we can get $C_{1}(0)=-26.2632-7.5707 i$, then $\mu_{2}=12.1471, \beta_{2}=-52.5264, T_{2}=5.8586$. From Theorem 3.1, we know that the Hopf bifurcation is supercritical and the bifurcating periodic solutions are stable.

\section{Conclusion}

In this paper, a delayed predator-prey system with Beddington-DeAngelis functional response has been investigated. The bifurcation of a predator-prey system with single delay has been studied by many researchers [16, 27-30]. However, there are few papers considering the bifurcation of a predator-prey system with multiple delays (see [31-33]). Compared with the system considered in [16], the system in this paper accounts for not only the feedback delay of the prey density but also the feedback delay of the predator. The sufficient conditions for the stability of the positive equilibrium and the existence of periodic solutions 

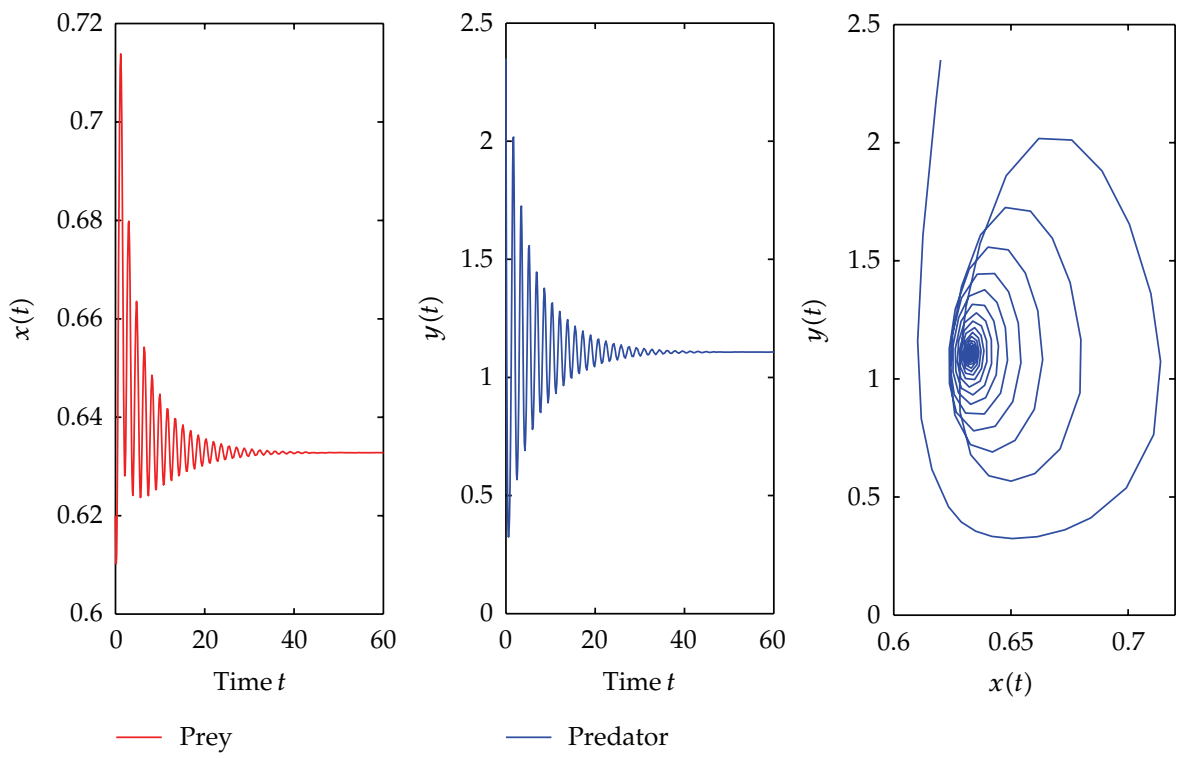

Figure 1: $E_{*}$ is stable for $\tau_{1}=\tau_{2}=\tau=0.4<\tau_{0}=0.4292$ with initial value $0.62,2.35$.
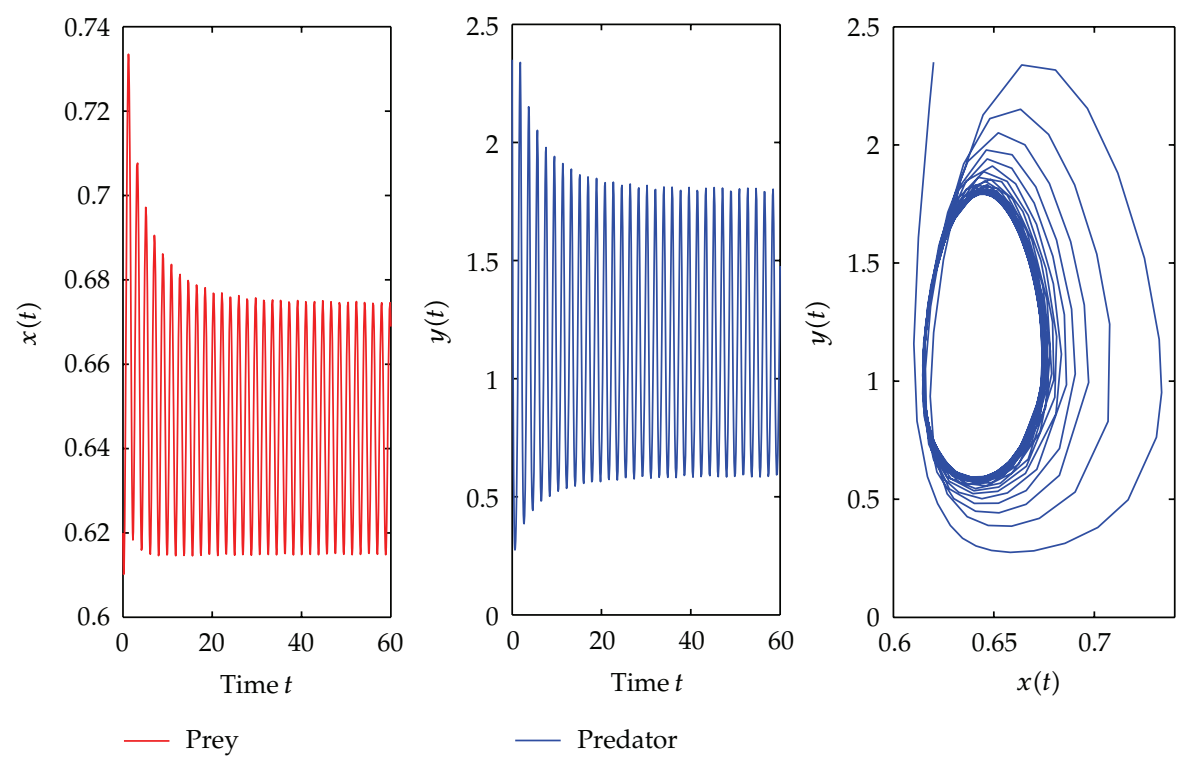

Figure 2: $E_{*}$ is unstable for $\tau_{1}=\tau_{2}=\tau=0.44>\tau_{0}=0.4292$ with initial value 0.62,2.35.

via Hopf bifurcation at the positive equilibrium are obtained when $\tau_{1}=\tau_{2}$ and $\tau_{1} \neq \tau_{2}$ with $\tau_{2} \in\left[0, \tau_{20}\right)$. Special attention is paid to the direction of the Hopf bifurcation and the stability of the bifurcating periodic solutions. By computation, we find that the feedback delay of the predator is marked because the critical value of $\tau_{2}$ is much smaller when we only consider it. The feedback delay of the prey is unremarkable because the critical value of $\tau_{1}$ is much bigger when we consider it with $\tau_{2}$ in its stable interval. Furthermore, Zhang [16] has obtained that the two species in system (1.4) with only the feedback delay of the predator could coexist. 

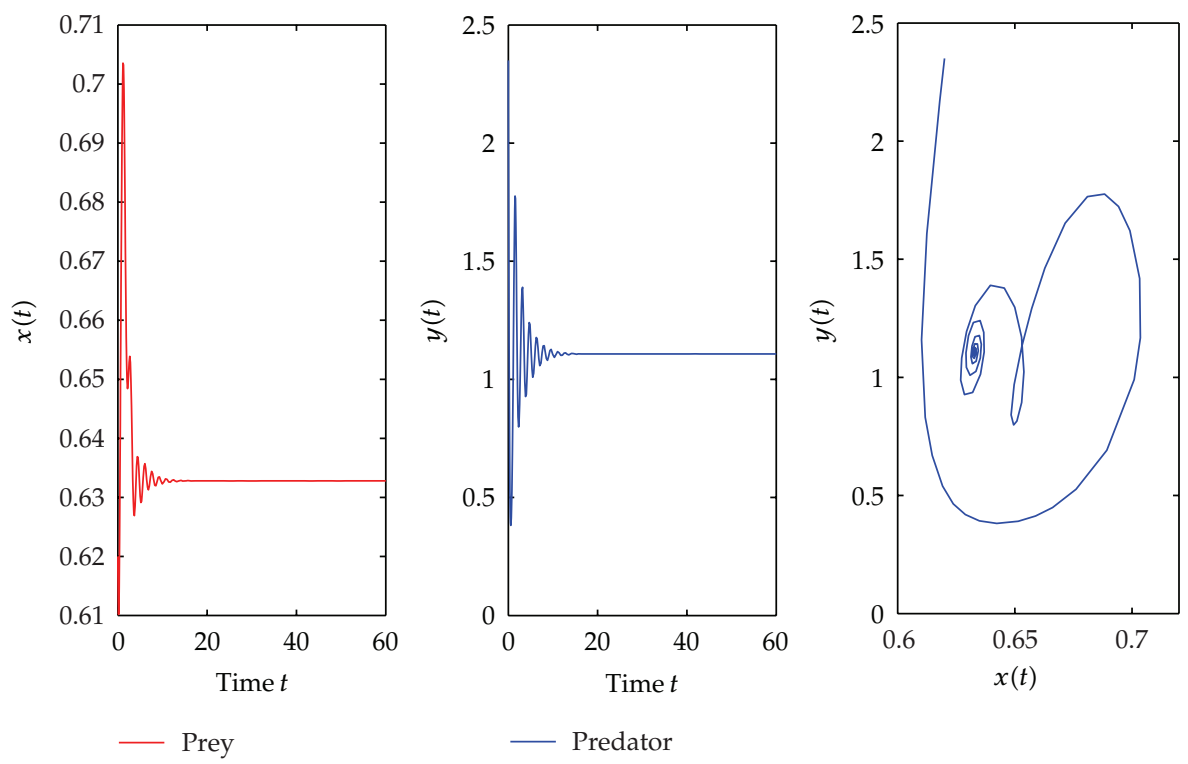

Figure 3: $E_{*}$ is stable for $\tau_{1}=0.8<\tau_{1} *=2.3211, \tau_{2}=0.36$ with initial value 0.62,2.35.
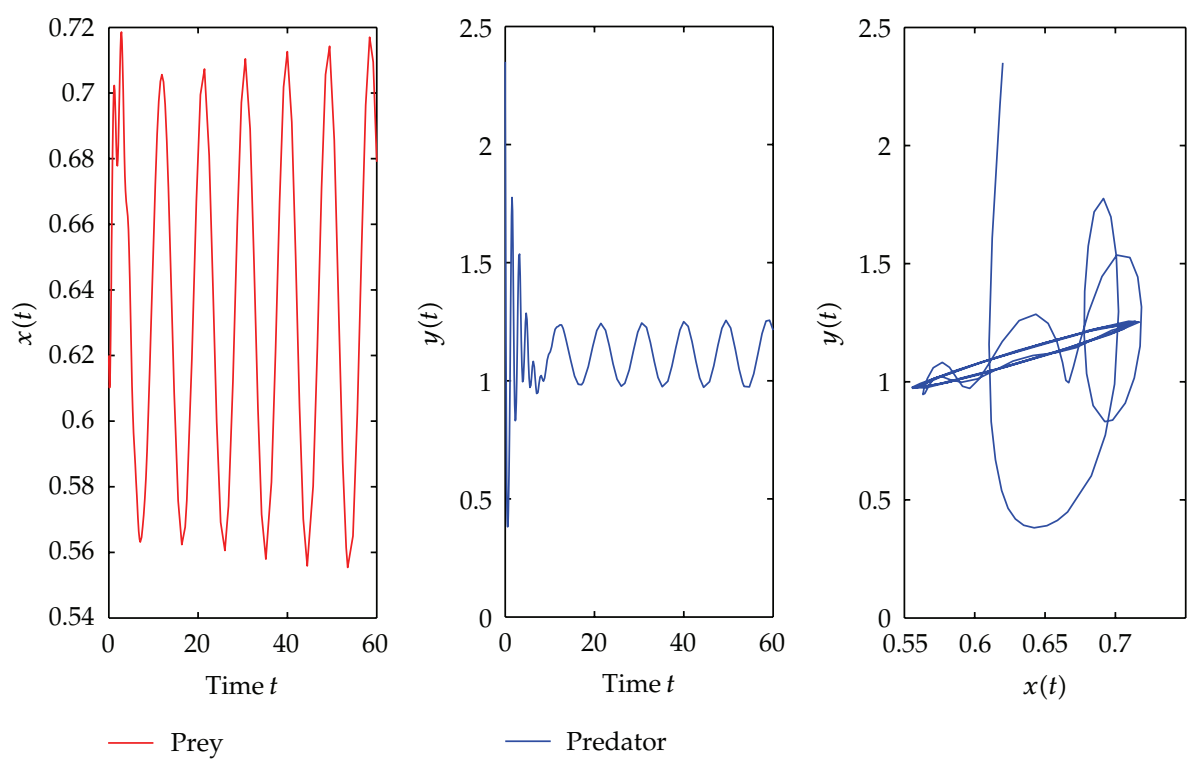

Figure 4: $E_{*}$ is unstable for $\tau_{1}=2.4>\tau_{1} *=2.3211, \tau_{2}=0.36$ with initial value 0.62,2.35.

However, we get that the two species could also coexist with some available feedback delays of the prey and the predator. This is valuable from the view of ecology.

Unfortunately, the existence of the periodic solutions remain valid only in a small neighborhood of the critical value. It is definitely an interesting work to investigate whether these nonconstant periodic solutions which are obtained through local Hopf bifurcation can still exist for large values of the corresponding parameter time delay. The global continuation of the local Hopf bifurcation is left as the future work. 


\section{Acknowledgments}

The authors are grateful to the two anonymous reviewers for their helpful comments and valuable suggestions on improving the paper. This work was supported by Doctor Candidate Foundation of Jiangnan University (JUDCF12030) and Anhui Provincial Natural Science Foundation under Grant no. 1208085QA11.

\section{References}

[1] E. Beretta and Y. Kuang, "Global analyses in some delayed ratio-dependent predator-prey systems," Nonlinear Analysis: Theory, Methods E Applications, vol. 32, no. 3, pp. 381-408, 1998.

[2] A. A. Berryman, "The origins and evolution of predator-prey theory," Ecology, vol. 73, no. 5, pp. 1530 1535, 1992.

[3] H. J. Guo and X. X. Chen, "Existence and global attractivity of positive periodic solution for a Volterra model with mutual interference and Beddington-DeAngelis functional response," Applied Mathematics and Computation, vol. 217, no. 12, pp. 5830-5837, 2011.

[4] X. L. Wang, Z. J. Du, and J. Liang, "Existence and global attractivity of positive periodic solution to a Lotka-Volterra model," Nonlinear Analysis: Real World Applications, vol. 11, no. 5, pp. 4054-4061, 2010.

[5] Z. D. Teng, "On the persistence and positive periodic solution for planar competing Lotka-Volterra systems," Annals of Differential Equations, vol. 13, no. 3, pp. 275-286, 1997.

[6] P. H. Leslie, "Some further notes on the use of matrices in population mathematics," Biometrika, vol. 35 , no. 3-4, pp. 213-245, 1948.

[7] M. A. Aziz-Alaoui and M. D. Okiye, "Boundedness and global stability for a predator-prey model with modified Leslie-Gower and Holling-type II schemes," Applied Mathematics Letters, vol. 16, no. 7, pp. 1069-1075, 2003.

[8] Z. Zhao, L. Yang, and L. Chen, "Impulsive perturbations of a predator-prey system with modified Leslie-Gower and Holling type II schemes," Journal of Applied Mathematics and Computing, vol. 35, no. 1-2, pp. 119-134, 2011.

[9] S. Gakkhar and A. Singh, "Complex dynamics in a prey predator system with multiple delays," Communications in Nonlinear Science and Numerical Simulation, vol. 17, no. 2, pp. 914-929, 2012.

[10] G. O. Eduardo, M. L. Jaime, A. Rojas-Palma, and J. D. Flores, "Dynamical complexities in the Leslie-Gower predator-prey model as consequences of the Allee effect on prey," Applied Mathematical Modelling, vol. 35, no. 1, pp. 366-381, 2011.

[11] J. T. Tanner, "The stability and intrinsic growth rates of prey and predator populations," Ecology, vol. 56, no. 4, pp. 855-867, 1975.

[12] A. Gasull, R. E. Kooij, and J. Torregrosa, "Limit cycles in the Holling-Tanner model," Publicacions Matemàtiques, vol. 41, no. 1, pp. 149-167, 1997.

[13] E. Sáez and E. G. Olivares, "Dynamics of a predator-prey model," SIAM Journal on Applied Mathematics, vol. 59, no. 5, pp. 1867-1878, 1999.

[14] T. Saha and C. G. Chakrabarti, "Dynamical analysis of a delayed ratio-dependent Holling-Tanner predator-prey model," Journal of Mathematical Analysis and Applications, vol. 358, no. 2, pp. 389-402, 2009.

[15] K. Q. Lan and C. R. Zhu, "Phase portraits, Hopf bifurcations and limit cycles of the Holling-Tanner models for predator-prey interactions," Nonlinear Analysis: Real World Applications, vol. 12, no. 4, pp. 1961-1973, 2011.

[16] J.-F. Zhang, "Bifurcation analysis of a modified Holling-Tanner predator-prey model with time delay," Applied Mathematical Modelling, vol. 36, no. 3, pp. 1219-1231, 2012.

[17] J. R. Beddington, "Mutual interference between parasites or predators and its effect on searching efficiency," Journal of Animal Ecology, vol. 44, no. 1, pp. 331-340, 1975.

[18] D. L. DeAngelis, R. A. Goldstein, and R. V. ONeill, "A model for trophic interaction," Ecology, vol. 56, no. 4, pp. 881-892, 1975.

[19] T.-W. Hwang, "Global analysis of the predator-prey system with Beddington-DeAngelis functional response," Journal of Mathematical Analysis and Applications, vol. 281, no. 1, pp. 395-401, 2003.

[20] M. Fan and Y. Kuang, "Dynamics of a nonautonomous predator-prey system with the BeddingtonDeAngelis functional response," Journal of Mathematical Analysis and Applications, vol. 295, no. 1, pp. 15-39, 2004. 
[21] K. Q. Lan and C. R. Zhu, "Phase portraits of predator-prey systems with harvesting rates," Discrete and Continuous Dynamical Systems A, vol. 32, no. 3, pp. 901-933, 2012.

[22] Z. Q. Lu and X. Liu, "Analysis of a predator-prey model with modified Holling-Tanner functional response and time delay," Nonlinear Analysis: Real World Applications, vol. 9, no. 2, pp. 641-650, 2008.

[23] S. G. Ruan and J. J. Wei, "On the zeros of transcendental functions with applications to stability of delay differential equations with two delays," Dynamics of Continuous, Discrete $\mathcal{E}$ Impulsive Systems A, vol. 10, no. 6, pp. 863-874, 2003.

[24] Y. Kuang, Delay Differential Equations with Applications in Population Dynamics, vol. 191, Academic Press, New York, NY, USA, 1993.

[25] J. K. Hale, Theory of Functional Differential Equations, Springer, New York, NY, USA, 1977.

[26] B. D. Hassard, N. D. Kazarinoff, and Y. H. Wan, Theory and Applications of Hopf Bifurcation, Cambridge University Press, Cambridge, UK, 1981.

[27] L. Feng and L. H. Wei, "Hopf bifurcation of a predator-prey model with time delay and stage structure for the prey," Mathematical and Computer Modelling, vol. 55, no. 3-4, pp. 672-679, 2012.

[28] Y. Yang, "Hopf bifurcation in a two-competitor, one-prey system with time delay," Applied Mathematics and Computation, vol. 214, no. 1, pp. 228-235, 2009.

[29] Y. L. Song, S. L. Yuan, and J. M. Zhang, "Bifurcation analysis in the delayed Leslie-Gower predatorprey system," Applied Mathematical Modelling, vol. 33, no. 11, pp. 4049-4061, 2009.

[30] S. Yuan and Y. Song, "Stability and Hopf bifurcations in a delayed Leslie-Gower predator-prey system," Journal of Mathematical Analysis and Applications, vol. 355, no. 1, pp. 82-100, 2009.

[31] Y. F. Ma, "Global Hopf bifurcation in the Leslie-Gower predator-prey model with two delays," Nonlinear Analysis: Real World Applications, vol. 13, no. 1, pp. 370-375, 2012.

[32] X.-Y. Meng, H.-F. Huo, and H. Xiang, "Hopf bifurcation in a three-species system with delays," Journal of Applied Mathematics and Computing, vol. 35, no. 1-2, pp. 635-661, 2011.

[33] S. Gakkhar, K. Negi, and S. K. Sahani, "Effects of seasonal growth on ratio dependent delayed prey predator system," Communications in Nonlinear Science and Numerical Simulation, vol. 14, no. 3, pp. 850-862, 2009. 


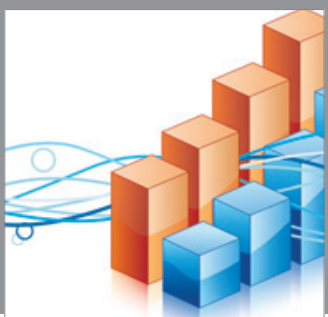

Advances in

Operations Research

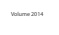

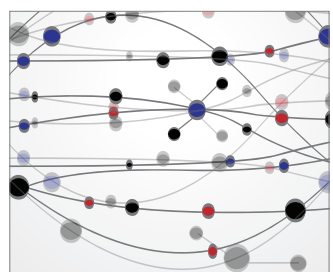

\section{The Scientific} World Journal
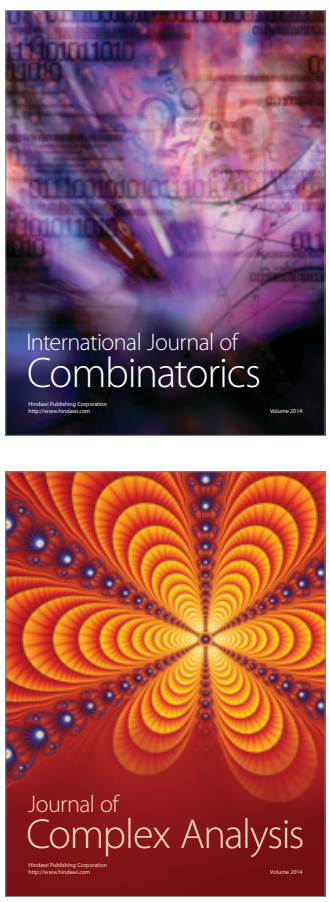

International Journal of

Mathematics and

Mathematical

Sciences
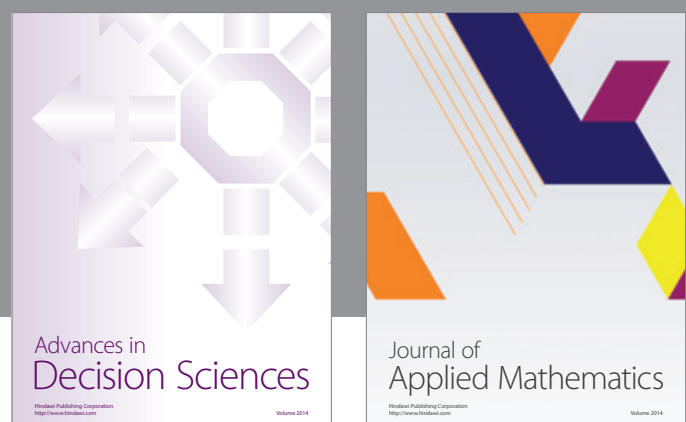

Journal of

Applied Mathematics
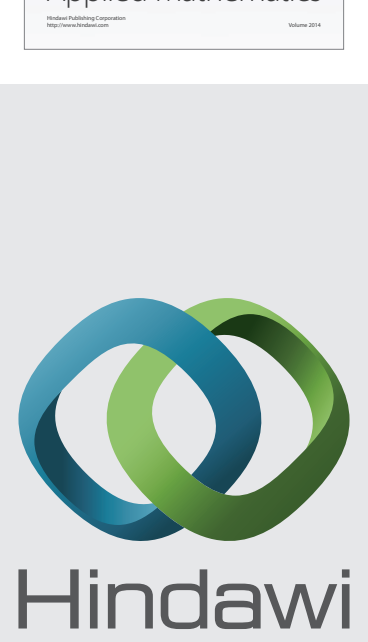

Submit your manuscripts at http://www.hindawi.com
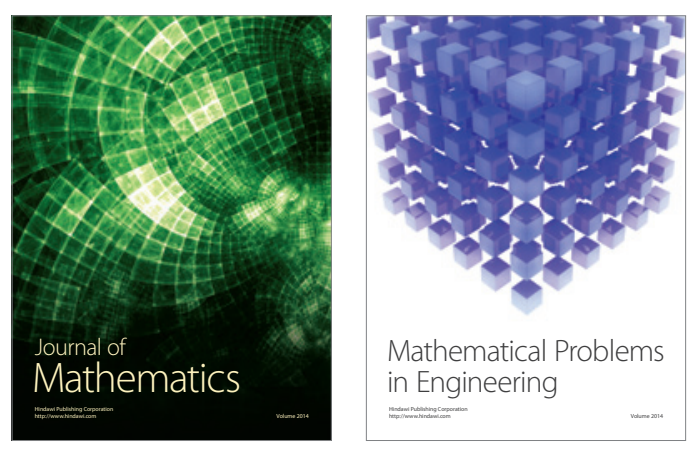

Mathematical Problems in Engineering
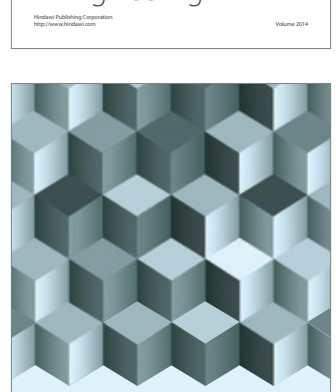

Journal of

Function Spaces
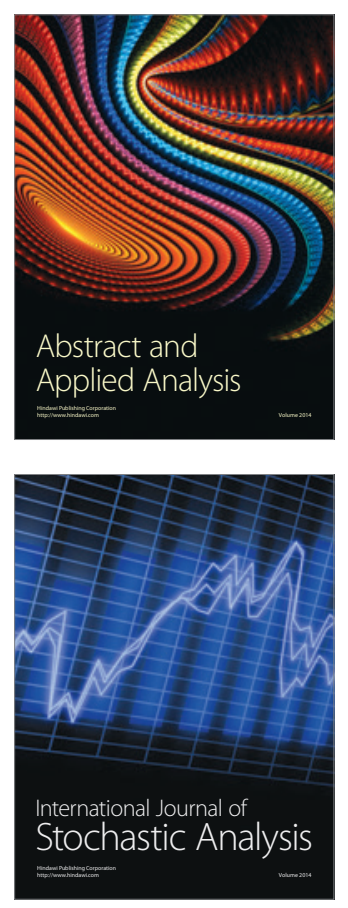

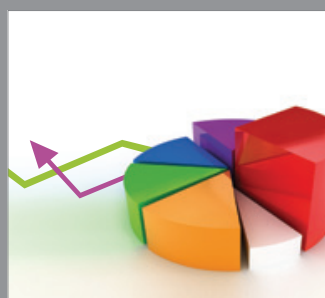

ournal of

Probability and Statistics

Promensencen
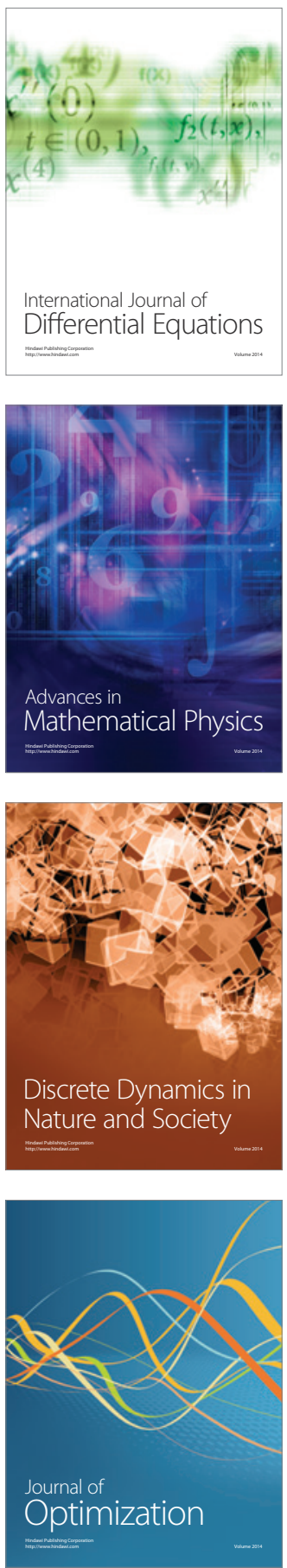